\title{
Applications of the Magnetocaloric Effect in Single-Stage, Multi-Stage and Continuous Adiabatic Demagnetization Refrigerators
}

Peter J Shirron

NASA/Goddard Space Flight Center, Greenbelt, MD 20771 USA

\begin{abstract}
Adiabatic demagnetization refrigerators (ADR), based on the magnetocaloric effect, are solid-state coolers that were the first to achieve cooling well into the sub-kelvin regime. Although supplanted by more powerful dilution refrigerators in the 1960s, ADRs have experienced a revival due to the needs of the space community for cooling astronomical instruments and detectors to temperatures below $100 \mathrm{mK}$. The earliest of these were single-stage refrigerators using superfluid helium as a heat sink. Their modest cooling power $(<1 \mu \mathrm{W}$ at $60 \mathrm{mK}[1])$ was sufficient for the small (6x6) detector arrays[2], but recent advances in arraying and multiplexing technologies[3] are generating a need for higher cooling power $(5-10 \mu \mathrm{W})$, and lower temperature $(<30 \mathrm{mK})$. Single-stage ADRs have both practical and fundamental limits to their operating range, as mass grows very rapidly as the operating range is expanded. This has led to the development of new architectures that introduce multi-staging as a way to improve operating range, efficiency and cooling power. Multi-staging also enables ADRs to be configured for continuous operation, which greatly improves cooling power per unit mass. This paper reviews the current field of adiabatic demagnetization refrigeration, beginning with a description of the magnetocaloric effect and its application in single-stage systems, and then describing the challenges and capabilities of multi-stage and continuous ADRs.
\end{abstract}

\section{INTRODUCTION}

Low temperature refrigeration is increasingly important to a growing number of scientific efforts, stemming in part from recent advances in low temperature detector technologies, but also from breakthrough developments in areas such as quantum computing. This growth is fueling the development of progressively more capable refrigerators, in the direction of lower temperature operation and higher cooling power. In addition, the rising cost of liquid helium is causing a widespread transition to the use of mechanical cryocoolers as the primary cooling system in both laboratory and space-based cryogenic systems. The challenge for low temperature refrigerators is to accommodate the higher base temperature and lower cooling power than was available from liquid helium. 
Among the refrigeration technologies that can be used in the temperature ranges of interest - from several kelvin to the deep sub-kelvin regime - adiabatic demagnetization refrigerators (ADR) have many advantages that are prompting more widespread use. Based on the magnetocaloric effect, which was first observed by Langevin[4] in 1905 and proposed as the basis for refrigeration by Debye[5], ADRs are compact, robust coolers that can operate over a wide temperature range with unmatched thermodynamic efficiency. The earliest implementation, by Giauque and McDougall in the 1930s[6], was also the first to demonstrate cooling to sub-kelvin temperatures. The technique was expanded variously over the next few decades, including the use of multiple stages (Darby[7]) and quasicontinuous stages (Heer, et al.[8]), but these systems were hampered by the difficulty of producing the large magnetic fields required. As a result, the development of dilution refrigeration in the 1960s, with higher cooling power and continuous operation, led to a steep decline in the use of magnetic coolers.

The revival of ADRs in the 1980s traces its origin to the development of low temperature detectors for astronomy applications[9]. These were thermal detectors which benefited from the reduced heat capacity associated with cooling to (initially) $\sim 1 \mathrm{~K}$ temperatures, then to temperatures in the 50-100 $\mathrm{mK}$ range. In the interim, magnet technology advanced markedly, both in terms of engineering current density and small wire size. The commercial availability of small diameter, multifilamentary wire makes it possible construct compact, high field (2-4 T), low current (2-4 amps) magnets that suitable for use in space missions using ADRs[10,11], as well as low cost laboratory systems.

For space applications, ADRs have many distinct advantages, including no gravity dependence, very high thermodynamic efficiency, and an ability to control temperature without dissipating heat. The high efficiency is due to the reversible nature of the magnetocaloric effect, and is important in reducing the cooling requirements for the cryogenic system that acts as the ADR's heat sink. Ultimately this reduces the size, mass and power requirements for the instrument. Low temperature detectors tend to require very stable and precise temperature control - often at the level of $1 \mu \mathrm{K} \mathrm{rms}$ or better - and the unique relationship between magnetic field and temperature allows temperature to be regulated at any value of interest by feedback control of the ADR's magnet current[19, 20]. Non-dissipative temperature control improves efficiency by maximizing the availability of the ADR's cooling capacity for absorbing instrument heat loads.

At the same time that ADR technology was advancing, significant progress was being made in detector technologies, fabrication techniques and arraying capabilities, resulting in vastly improved resolution and expanded array sizes, with corresponding increases in heat loads and dissipation. The consequence is a need for more capable refrigerators, in terms of both cooling power and lower operating temperature. To meet these needs, different ADR architectures are being proposed and implemented, tending now toward multi-stage configurations[12] in order to achieve wider operating range, lower heat loads on the coldest stages, lower overall mass, and even continuous operation[13], which itself significantly improves cooling power and efficiency, and reduces size and mass.

The field is rapidly expanding, under a demand for coolers that are tailored to highly specific instrument requirements, particularly space instruments, and for coolers suited to 
multi-purpose facilities. This paper summarizes the progress that has been made in recent years, particularly in multi-stage units, to improve the performance and utility of ADRs.

\section{MAGNETOCALORIC EFFECT}

At it most basic level, the magnetocaloric effect is a phenomenon in which certain materials warm or cool as they are exposed to increasing or decreasing magnetic fields. In paramagnetic materials, the effect originates in the interaction of an external magnetic field with the magnetic moment of unpaired outer-shell electrons, and results in the entropy having a strong dependence on magnetic field and temperature. Detailed discussions of the magnetocaloric effect may be found in the literature $[14,15]$. Conveniently, the entropy of a system of $n$ (in moles) magnetic moments characterized by the quantum number $J$, at temperature $T$ and in a magnetic field $B_{e f f}$, can be expressed in analytic form:

$$
\begin{array}{r}
S / R=n\left(x \operatorname{coth}(x)-(2 J+1) x \operatorname{coth}((2 J+1) x)+\ln \left(\frac{\sinh ((2 J+1) x)}{\sinh (x)}\right)\right) \\
x \equiv \mu_{B} g B_{e f f} / 2 k_{B} T=(0.336 \mathrm{~K} / \mathrm{T}) g B_{e f f} / T
\end{array}
$$

where $\mu_{B}$ is the Bohr magneton, $k_{B}$ is Boltzmann's constant, and $g$ is the Landé g-factor for the magnetic ion. The factor $2 J+1$ represents the number of possible values for the zcomponent of the magnetic moment. In the case of an "ideal" paramagnet consisting of non-interacting moments, these states are degenerate, and the magnetic field, $B_{e f f}$, is simply the externally applied field. Thus we find that in zero magnetic field, the entropy per mole is $R \ln (2 J+1)$.

In a real paramagnet, however, the magnetic moments interact with each other and/or the crystal field. At low enough temperature, the interaction energy causes the moments to self-align, resulting in a suppression of the entropy. In the limit of $T \rightarrow 0 \mathrm{~K}$, the entropy is suppressed to zero.

It has become common to account for these interactions by defining a background field, $b$, that is rms averaged with the applied field, $B$, such that:

$B_{e f f}=\sqrt{B^{2}+b^{2}}$

In practice, using a constant value for $b$ will reproduce the general temperature dependence of entropy, but for purposes of optimizing "salt pills" (the structure that contains the magnetic refrigerant in an ADR), a more accurate representation is often needed. From measurements of entropy at zero field, for example, one can compute values of $b$ that exactly reproduce the temperature dependence of the entropy. The functional form is difficult to justify on theoretical grounds, but we have found that an exponential of the form

$b(T)=b_{0}\left(1-e^{-\left(T / T_{0}\right)^{a}}\right)$ 
can reproduce the zero field entropy over a wide temperature range $(0-5 \mathrm{~K})$ to within a few percent.

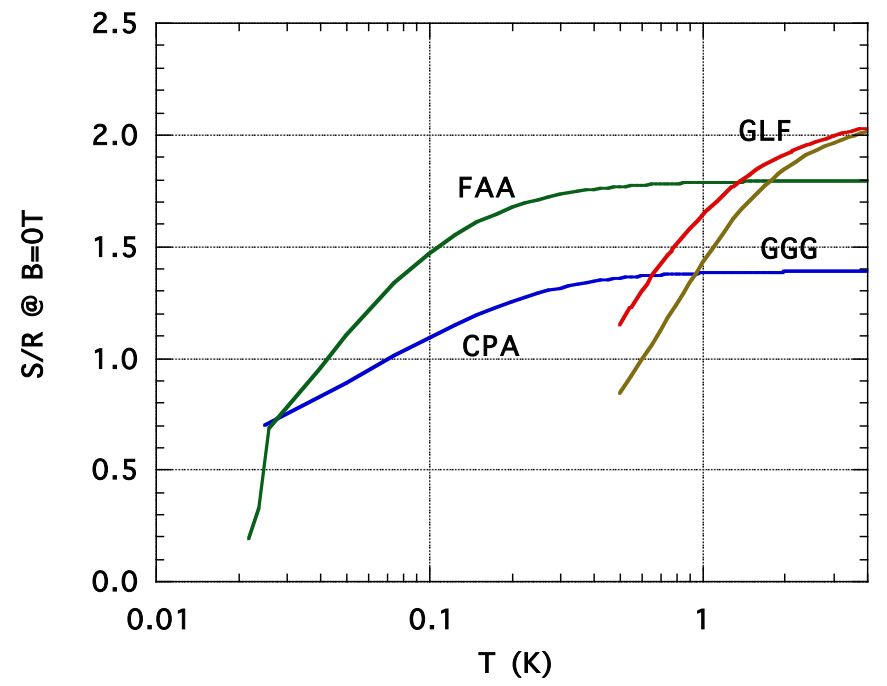

FIGURE 1: Zero-field entropies for common magnetocaloric materials used in low temperature ADRs. [16, 17, 18]

Figure 1 shows the zero-field entropy curves for some of the most common magnetic refrigerants use in ADRs, and Table 1 summarizes their relevant parameters, including coefficients for $b(T)$. The Neel temperature, at which the material enters an ordered magnetic state, shows a clear correlation with $J$ and magnetic ion density. A stronger magnetic moment (higher $\int$ ) and closer ion spacing (higher density) both tend to strengthen nearest neighbor interactions, and raise the temperature at which internal interactions begin to affect the entropy. The upshot is that low temperature refrigerants must be relatively dilute and have lower spin. As a consequence, they have relatively low entropy (or heat) storage capacity, and as lower temperature systems are built, increasing care must be exercised to limit the heat loads reaching the coldest stage.

Table 1. Selected parameters for common magnetocaloric materials

\begin{tabular}{|l|l|l|l|l|l|l|l|}
\hline Material & $\mathrm{J}$ & $\begin{array}{l}\text { Magnetic ion } \\
\text { density }(/ \mathrm{cm} 3)\end{array}$ & $\begin{array}{l}\text { Neel } \\
\text { temperature } \\
(\mathrm{K})\end{array}$ & $\begin{array}{l}\mathrm{b} \\
(\text { Tesla })\end{array}$ & $\begin{array}{l}\mathrm{b}_{0} \\
(\text { Tesla })\end{array}$ & $\mathrm{T}_{0}(\mathrm{~K})$ & $\mathrm{a}$ \\
\hline CPA & $3 / 2$ & $2.14 \times 10^{21}$ & 0.009 & & 0.0841 & 0.0836 & 0.7973 \\
\hline FAA & $5 / 2$ & $2.15 \times 10^{21}$ & 0.026 & & 0.0560 & 0.0601 & 0.3770 \\
\hline GGG & $7 / 2$ & $1.27 \times 10^{22}$ & 0.38 & 0.481 & & & \\
\hline GLF & $7 / 2$ & $1.43 \times 10^{22}$ & $<0.25$ & 0.38 & & & \\
\hline
\end{tabular}


ADR Cycle - Single-stage System

From Eq. 2, we see one of the most important features of the magnetocaloric effect in paramagnets: the entropy depends only on the ratio of magnetic field and temperature, $B_{\text {eff }} / T$. Under adiabatic conditions, a change in magnetic field produces a proportional change in temperature. Equally important, the change in temperature is completely reversible and involves no dissipative processes. It is therefore straightforward to construct a refrigeration cycle based on the magnetocaloric effect, with relatively few components, that is very robust and able to achieve very high efficiency.

To illustrate the cycle used in ADRs, Figure 2 shows entropies for ferric ammonium alum (FAA) at zero-field and at 1, 2, and 3 T. Superimposed are the steps of an ideal ADR cycle.

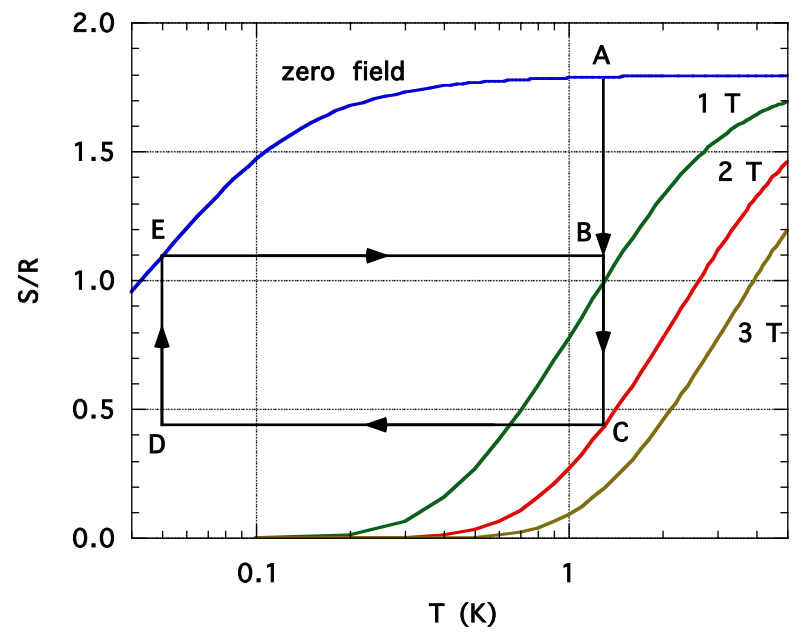

FIGURE 2: Entropy diagram for FAA.

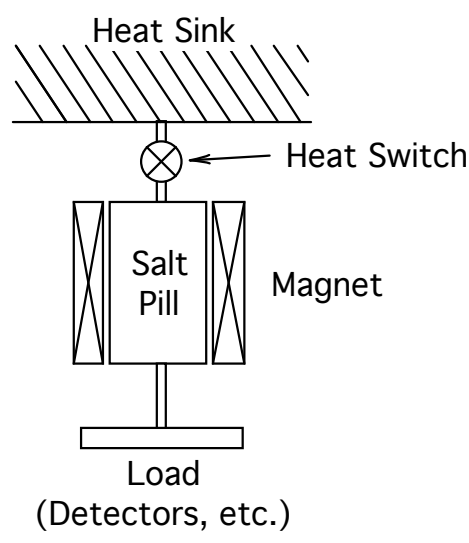

FIGURE 3: Schematic of a single-stage ADR. 

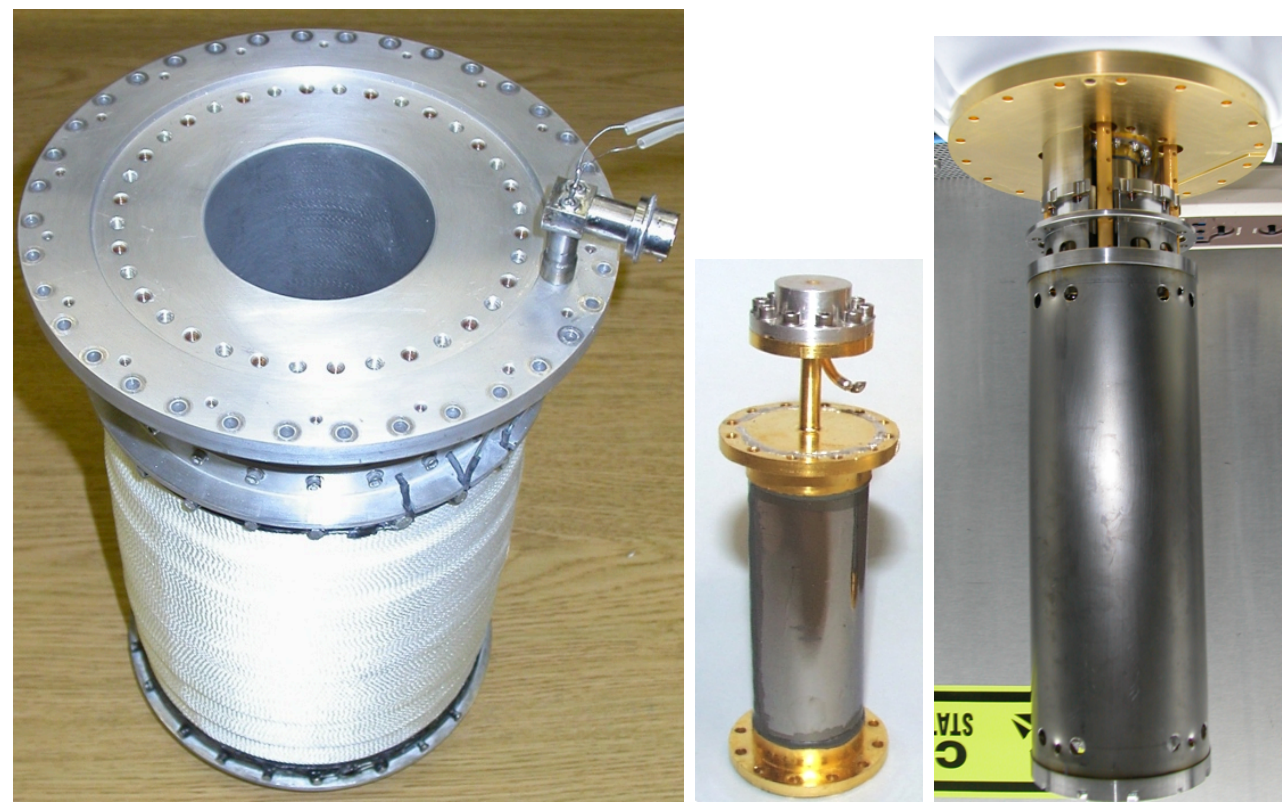

Figure 4. Components of the Suzaku ADR. At left is the immersion-cooled $2 \mathrm{amp} / 2 \mathrm{~T}$ magnet with an 8-cm diameter bore, middle is the gas-gap heat switch, and right is the salt pill/heat switch assembly containing 920 grams of FAA. The heat switch links the salt pill to the top plate, which bolts to the top of the magnet.

The standard ADR configuration is shown schematically in Figure 3, and in Figure 4 are the components produced for the Suzaku ADR[19]. The basic components are a salt pill located in the bore of a superconducting magnet, and a heat switch. Generally the heat switch is actively controlled to switch between high and low thermal conductance, but passive switches have also been successfully used[20]. Also required, but not shown, is some means of suspending the salt pill so that it is physically supported but thermally isolated from the magnet.

At the starting point, $\mathrm{A}$, the salt pill is at zero field and has been cooled to the temperature of a heat sink, $T_{\text {sink. }}$. The heat switch remains closed as the magnetic field is ramped up toward it maximum value. During this isothermal magnetization, entropy decreases as heat flow from the salt pill to the heat sink:

$\dot{Q}=T_{\text {sink }} \dot{S}=T_{\text {sink }} \frac{d S}{d B} \dot{B}$

Magnetization continues until full field, $B_{\max }$ is reached (C), at which point the heat switch is opened. The magnetic field is then reduced, adiabatically cooling the salt pill from the sink temperature until the load reaches its desired value, $T_{\text {load }}(\mathrm{D})$. Point $\mathrm{D}$ will be reached with some magnetic field remaining. As heat is absorbed at low temperature, the magnetic field can be decreased to maintain constant temperature. Eventually the field will be reduced to zero (E), at which point the ADR's cooling capacity is exhausted. 
The cycle can be repeated from point $\mathrm{E}$ by increasing the magnetic field to warm the salt pill above the heat sink (B), at which point the heat switch is closed, and cycle continues as before.

The hold time of the ADR (the period between D and E), depends on the entropy capacity that has been generated during magnetization, and the heat load, $\dot{Q}_{\text {load }}$ :

$$
t_{\text {hold }}=\frac{T_{\text {load }}}{\dot{Q}_{\text {load }}}\left(S\left(T_{\text {load }}, 0\right)-S\left(T_{\text {sink }}, B_{\text {max }}\right)\right)
$$

ADR stages can be designed for a wide variety of purposes, covering a wide range of temperatures, and a wide range of cooling capacities. The example that has been used in this discussion is the ADR that was flown on the Suzaku x-ray satellite, which used 920 grams of FAA, a peak magnetic field of $2 \mathrm{~T}$, and a $1.3 \mathrm{~K}$ helium bath as a heat sink, and operated at $60 \mathrm{mK}$. The theoretical maximum cooling capacity at $60 \mathrm{mK}$ is $0.67 \mathrm{~J}$, whereas the measured capacity was $0.45 \mathrm{~J}$. The hold time was required to be greater than 24 hours. With required margin on the original design loads that were predicted to be $3.0 \mu \mathrm{W}$, the ADR actually achieved hold times in excess of 36 hours.

\section{Temperature Control and Deterministic Operation}

Unlike other low temperature refrigeration techniques where the cooling mechanism is separate from temperature control, the temperature of an ADR stage can be precisely controlled through its magnetic field[21,22]. During the demagnetization phase, magnet current is reduced until the salt pill (or an attached load) is cooled to the desired operating temperature (which can be any value below the starting point). As heat is slowly absorbed, the magnetic field (or magnet current) can be reduced at a controlled rate to produce very stable temperature. Stability at the sub-microkelvin rms level is common.

At this point we should note that the magnetic field in the magnet is usually not uniform. Even complex winding geometries cannot achieve truly uniform fields. This might seem to complicate the temperature control issue, but since the magnetic field is everywhere proportional to current, and temperature changes are proportional to changes in field, the temperature will change uniformly across the salt pill. The exception to this is when the external field becomes comparable to or smaller than the background field, $b$. At that point, the strongest field regions will produce more cooling, and small temperature gradients will exist within the salt pill. This results in some inefficiency, but is essentially transparent to the control system.

Thus, in ADR operation, it has become common to use temperature as the input to a feedback loop whose output is either magnet current or magnet voltage. In the latter case, the output voltage, when using a 4-wire power supply, imposes a current ramp rate proportional to the inductance. Both forms of control have been successfully implemented[23, 24], but voltage control has the advantage of requiring much lower bandwidth to achieve stable temperature control, since the demagnetization voltage for a fixed heat load is at most a very slowly varying function of time. 
Regardless of which method is used, the current in the magnet follows a predictable curve. Assuming a constant heat load, the salt pill's entropy increases at a fixed rate. Since entropy is dependent only on magnetic field and temperature (along with other fixed quantities such as refrigerant mass), the magnetic field can readily be calculated as a function of time. This has a number of advantages, such as the ability to predict the remaining hold time, or, by fitting to calculated demagnetization curves, to determine the salt temperature and entropy absorption rate. Knowledge of these parameters can be used to determine the efficiency of heat absorption.

As an example, Figure 5 shows a demagnetization curve for CPA controlled at $T_{\text {salt }}=50 \mathrm{mK}$. The horizontal axis is the stored cooling capacity per mole of refrigerant, equal to $T_{\text {salt }} \bullet\left(S\left(0, T_{\text {salt }}\right)-S\left(B, T_{\text {salt }}\right)\right) / n$, and scaled to units of $\mu \mathrm{W} \bullet$ hours $/ \mathrm{mol}$. Multiplying this quantity by the refrigerant mass and dividing by heat load yields a plot of magnetic field as a function of time (with time counting down to zero), or, conversely, the remaining hold time as a function of magnetic field.

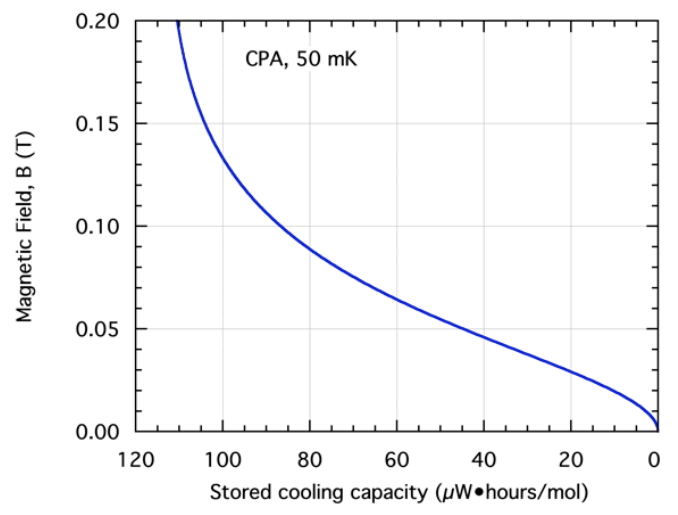

Figure 5. The magnetic field as a function of stored cooling capacity of a CPA stage at 50 $\mathrm{mK}$ can be used to calculate hold time or a "standard" demagnetization curve.

Another advantage of the deterministic nature of ADRs is that one can easily calculate the rate at which a stage must be magnetized or demagnetized to maintain temperature or to establish a desired cooling (or heating) rate. For example, the demagnetization rate needed to compensate for an applied heat load, $\dot{Q}$, is:

$\frac{d B}{d t}=-\frac{\dot{Q}}{\left(n_{\text {salt }} C\right) T_{\text {salt }}} \frac{B^{2}+b^{2}}{B}$

where

$C / R=\left(\frac{x^{2}}{\sinh ^{2}(x)}-\frac{(2 J+1)^{2} x^{2}}{\sinh ^{2}((2 J+1) x)}\right)$ 
with $x$ defined as in Eqn. 1. Here we have taken $b$ to be a constant. In the more general case, its temperature dependence must be included in the calculation of $C=T \frac{d S}{d T}=T \frac{d S}{d x} \frac{d x}{d T}$.

In principle, temperature control can be improved in cases where the instantaneous heat load, or changes in the heat load, can be known, since the compensating (de)magnetization rate can be applied in real time, and not based on the feedback control system's response to a temperature error.

\section{Inefficiencies}

ADR operation, so far, has been discussed in the context of an idealized cycle. In a practical system, there are inefficiencies that reduce the realizable cooling capacity. A more realistic ADR cycle is depicted in Figure 6. During isothermal magnetization (B-C), the finite thermal conductances of the heat switch and thermal bus that connects the magnetocaloric material to the heat switch give rise to a gradient between the salt and the heat sink. In situations where magnetization must occur within a prescribed time period in order to meet recycle time requirements, the gradient can be a substantial fraction of $T_{\text {sink. }}$. Since the elevated salt temperature increases the total amount of heat rejected to the heat sink, by the ratio $T_{\text {salt }} / T_{\text {sink, }}$, the type and design of the heat switch and thermal bus are among the most important considerations in system design.

After full field is reached, the salt pill will cool $\left(C^{\prime}-C\right)$ along the entropy curve for constant field, exponentially approaching $T_{\text {sink. }}$. In practice, the salt pill will be thermally isolated (by opening the heat switch) at some temperature above $T_{\operatorname{sink}}$, at which point it has a higher starting entropy than in the ideal cycle, yielding a lower entropy capacity for the complete cycle.

During adiabatic demagnetization, additional losses of entropy capacity will result from 1) heat capacities that must be cooled to low temperature, especially if they lag in temperature, 2) eddy current dissipation in metallic structures used in the salt pill, and 3) temperature gradients within the salt pill.

Below $1 \mathrm{~K}$, most materials have very small heat capacity compared to that of the salt, and substantial masses can be cooled with little negative effect. There are two caveats here. The first is that cooling rates must be slower than the thermal time constant of the attached heat capacities, or the lag in temperature will cause excessive entropy generation as they equilibrate to low temperature. The second is that, due to a Schottky anomaly, stainless steels have an relatively large heat capacity that actually begins to rise below $0.1 \mathrm{~K}[26]$. Consequently, salt pill design[10,27,28] generally strives to minimize the amount of stainless steel, and, if used, to ensure that it is well thermally connected to the salt. The latter is often a challenge due to their relatively poor thermal conductivity. 


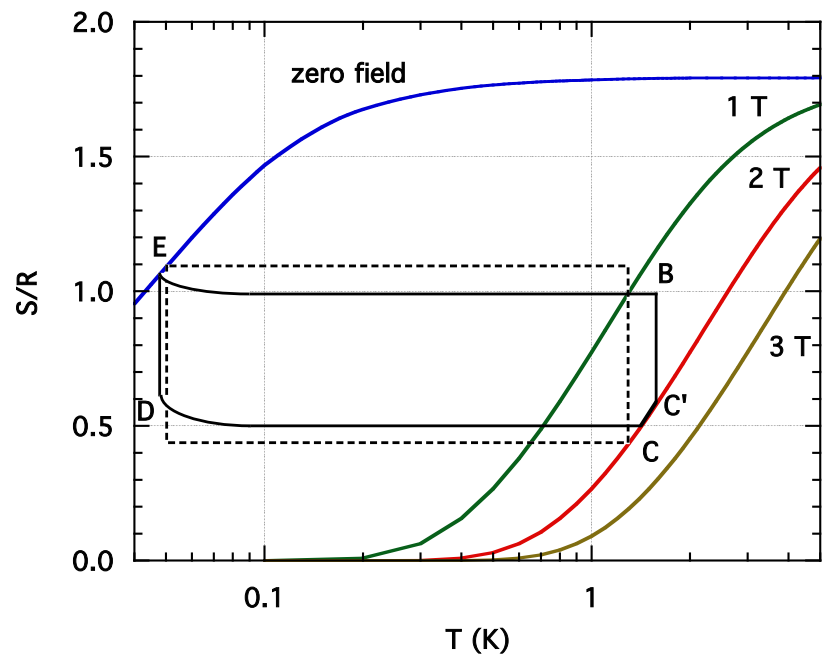

Figure 6. Realistic ADR cycle (solid lines) compared to ideal cycle (dashed box)

Eddy currents are a potential source of loss that can be controlled by geometry. Although the demagnetization rates in an ADR are small $(\sim 10 \mathrm{mT} / \mathrm{sec}), \mathrm{cm}$-sized loops of low resistivity material could dissipate heat that would be overwhelm the cooling generated, especially in very high purity, annealed material. High-conductivity materials used in the salt pill are either finely divided (e.g. small diameter wires) or cut so that loop areas are minimized. Perhaps the most problematic structures are the thermal interfaces at the end(s) of the salt pill. These, fortunately, are at the lowest field regions, and by interrupting loops where possible, eddy current losses are generally less than $1 \%$ of the cooling capacity.

At low temperature, the structure that thermally connects the salt to the load is again a source of inefficiency, primarily due to the thermal boundary resistance between the magnetocaloric material and thermal bus that acts as its thermal interface. Especially below $100 \mathrm{mK}$, the $T^{-3}$ dependence of the boundary resistance can translate to salt pill temperatures many millikelvins below the load temperature, depending on the heat load and details of the salt pill design.

This impacts the operation in two important ways. First, absorbing heat at lower temperature increases entropy generation by the ratio $T_{\text {load }} / T_{\text {salt }}$. Second, the zero-field entropy is lower for the colder salt, yielding a lower entropy capacity for the cycle.

With the loss of cooling capacity during demagnetization, and the inefficiencies associated with heat absorption at low temperature, is not unusual for the realizable cooling capacity, especially when operating below $100 \mathrm{mK}$, to be only $70-80 \%$ of the ideal capacity. 
In a single-stage ADR, the salt pill must cycle between the heat sink and its operating point in a single demagnetization stroke. While the operating range is in principle unlimited, in reality it becomes impractical to expand the range beyond about a factor of 20-30 in the ratio of the heat sink and operating temperatures.

The first, more fundamental, limitation comes from the magnetic field needed to drive the cycle. In an optimized stage[29], the optimal magnetic field depends on both the heat sink temperature, $T_{\text {sink }}$, and the operating range of the stage $\left(T_{\operatorname{sink}} / T_{\text {low }}\right)$. As the operating range exceeds a factor of $\sim 30$, the required magnetic field rapidly increases beyond 2-3 $\mathrm{T}$. As the operating range increases to a factor of 60 or more, the use of fields in excess of 4- $6 \mathrm{~T}$ is unavoidable.

Fields of this size are not far from the fundamental limits of NbTi magnet technology. The critical field of a superconductor decreases at higher temperature as 1- $\left(T / T_{c}\right)^{2}$; for NbTi, $T_{c}=9.2 \mathrm{~K}$. At $4.2 \mathrm{~K}$, fields of up to $8 \mathrm{~T}$ are possible, but at, say, $6 \mathrm{~K}$, the maximum possible field drops to half that value. Thus as heat sink temperatures rise, the ability to generate the necessary fields rapidly diminishes.

A related problem is the mass associated with progressively larger magnets, especially those that are shielded with ferromagnetic material. The thickness of a shield needed to contain all of the flux returned outside the windings is proportional to the central field, and to the outer diameter of the magnet. Shield mass will therefore increase at least as the square of the peak magnetic field, becoming prohibitive for fields in excess of about $4 \mathrm{~T}$.

The second limitation is the strong temperature dependence of parasitic heat loads from components that thermally link the salt pill to the heat sink, including the heat switch and suspension structures. The conduction has a dependence of $T_{\text {sink }}{ }^{m}$ (assuming that $T_{\text {low }}{ }^{m}<<T_{\operatorname{sink}^{m}}{ }^{2}$, where $m$ is at least 2 , and may be above 4 . As the sink temperature increases, these parasitic loads eventually dominate the cooling capacity requirements. Since entropy usage at the hold temperature varies as $1 / T_{\text {low, }}$, the required salt mass (and volume) scales at least as $T_{\operatorname{sink}^{2}} / T_{\text {low }}$ - requiring an even larger magnet and shield to accommodate the larger salt pill.

Finally, the heat capacity of the load being cooled may also be an important consideration. As mentioned above, at temperatures below $1 \mathrm{~K}$ most materials have small enough heat capacity that, provided they are in good thermal contact to the refrigerant, relatively large masses can be cooled with minimal loss of cooling capacity. However, at higher temperatures, the rapidly increasing lattice component $\left(\sim T^{3}\right)$ of materials (including the refrigerant itself) can become appreciable, and the loss of cooling capacity during demagnetization can affect the salt pill size and mass. To give some sense of scale, the heat capacity of CPA with $B / T \sim 1$ Tesla/K is $0.012 \mathrm{~J} / \mathrm{g} \bullet \mathrm{K}$. At $5 \mathrm{~K}$, the heat capacities of stainless steel 304 [26] and copper are $0.0023 \mathrm{~J} / \mathrm{g} \bullet \mathrm{K}$ and $0.00015 \mathrm{~J} / \mathrm{g} \bullet \mathrm{K}$. The refrigerant still dominates, but by a margin that becomes smaller and smaller at higher heat sink temperature. 
Two-Stage and Multi-Stage ADRs

In order to significantly increase the operating range of an ADR, it is therefore advantageous, if not outright necessary, to expand into multi-stage configurations. The advantages are: 1) each stage cycles over only a fraction of the total operating range, thereby reducing the magnetic fields required, and 2) each upper stage can act as a guard to reduce the parasitic heat loads on the lower stage(s), 3) the upper stage(s) can use higher density refrigerants, reducing both the salt pill volume and magnet/shield mass needed for a given cooling capacity.

Figure 7 shows schematics of two 2-stage ADRs that have been developed. The first is a single-magnet design first proposed by Hagmann and Richards [30] for a 2-stage ADR for the SIRTF mission. The second is a two-magnet design that has been adopted by Hepburn [31] in a design for the International X-ray Observatory (IXO) mission, and more recently by Shirron [32] for the Soft X-ray Spectrometer instrument on Astro-H. Both the singleand two-magnet versions are capable of operating in the 50-100 $\mathrm{mK}$ range, using heat sinks at $4.2 \mathrm{~K}$ or even higher. The colder stages use FAA or CPA, and the upper stages use GGG, dysprosium gallium garnet (DGG), or GLF.
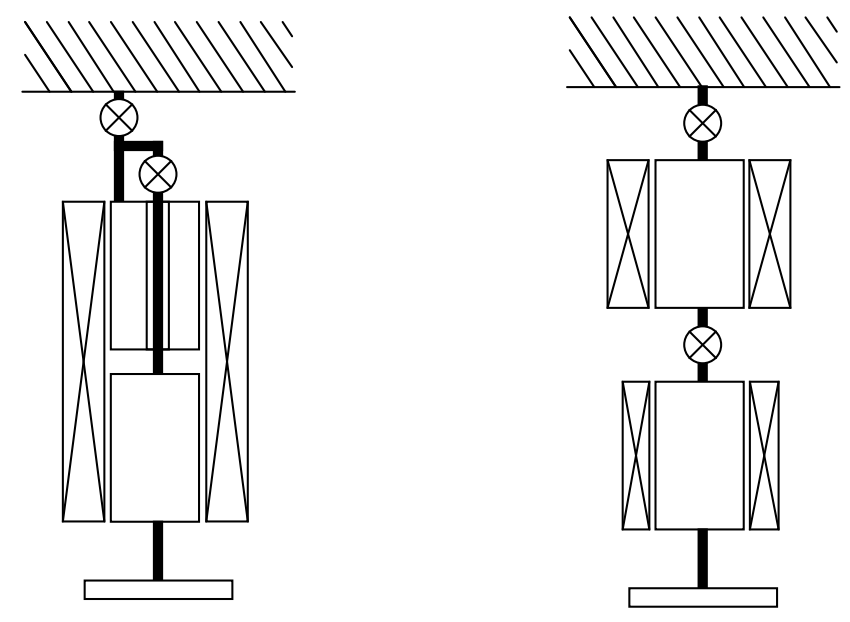

Figure 7. Schematics of 2-stage ADRs currently in use.

Single-Magnet Configuration - The single-magnet version is cycled in much the same way as a single-stage ADR: the magnetic field is ramped toward peak field, and as each salt pill warms above the heat sink, its heat switch is closed. After peak field is reached and the heat of magnetization has been transferred to the sink, the heat switches are opened, and the magnet is ramped down in field to cool the salt pills. The magnet current is regulated to stabilize the lower stage at tits operating point. The upper stage is not temperature controlled, but its high heat capacity results in a very slow drift during the hold. 
Since there is no heat exchange between stages, the amount of salt needed in each stage is dictated only by the parasitic heat loads during the hold time, and the cooling capacity per unit mass that is obtained for the chosen magnetic field, heat sink temperature and operating point. The main drawback is that the cold salt pill must still cycle across the full temperature range of the ADR, requiring relatively large magnetic fields (and magnetic shields, if used). And as mentioned above, depending on the heat capacity of the load, a significant portion of the cooling capacity may be lost on cooling to low temperature.

Two-Magnet Configuration - The two stages are controlled independently, with separate power supplies and temperature controllers, but in a coordinated manner to manage heat flow between them and to create the desired conditions for demagnetizing to low temperature. There are many recycling sequences that can be used, and these can have a significant impact on the salt masses and magnetic fields required. But the basic flow involves transferring heat from the lower stage to the upper at some intermediate temperature, $T_{1 \text { sink, }}$, then from the upper stage to the heat sink. During the hold period, both stages are temperature controlled (at $T_{1 l o w}$ and $T_{2 l o w}$ ), and, as in the single-magnet version, the upper stage acts as a guard to reduce the parasitic heat loads on the lower stage.

By cycling each stage over a portion of the ADR's temperature range, the magnetic field requirements for both stages are reduced. But since the upper stage must absorb all of the entropy stored by the lower stage during the cycle, its entropy capacity must be at least as large as that of the lower stage. The mass advantage, however, is still maintained since the upper stage can use much higher density materials.

Figure 8 shows an entropy diagram for a 2-stage ADR designed for operation at $50 \mathrm{mK}$ using a $4.2 \mathrm{~K}$ heat sink. In this case, $T_{1 \text { sink }}$ and $T_{2 l o w}$ are both shown to be $\sim 1 \mathrm{~K}$. In the design process, they are chosen to minimize cooling loads and, generally, overall mass. But it is important to keep in mind that they can be dynamically adjusted during operation to rebalance entropy capacities to accommodate changes in instrument heat loads or heat sink temperature.

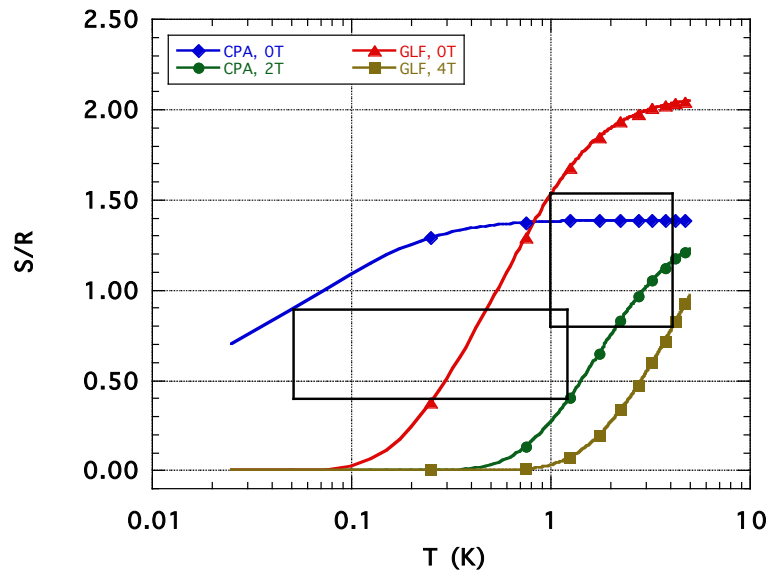

Figure 8. Entropy diagram for a 2-stage ADR operating between $50 \mathrm{mK}$ and $4.2 \mathrm{~K}$. 
Multi-Stage Configurations - There is, in principle, no limit to the number of stages that may be connected in series in a multi-stage ADR. The reasons for adding stages include extending the ADR's operating range, to add controlled temperature nodes for instrument components, such as amplifiers or optics, or to provide additional heat intercepts that reduce heat loads from components in both the ADR and whatever instrument is attached. Although increasing the number of stages adds complexity to the physical layout and control system, it can reduce mass and size, and improve overall efficiency through reductions in heat loads and magnetic field requirements.

For three or more stages, only the series configuration is practical. Entropy is flowed from stage to stage up to the heat sink, requiring each stage to have progressively higher entropy capacity. The sequence can start at the coldest stage, or at the warmest, in which case it can be thought of as a cascade of cooling capacity down to the cold end. The heat transfer points and hold temperatures are optimized using the same process as for two-stage systems.

\section{Continuous ADR}

While the ADR cycle is inherently single-shot - producing cooling for a period of time and then recycling at higher temperature - it can be adapted to continuous cooling. The primary benefit is that the need to store heat for long periods of time is eliminated, and since the cycling of an ADR stage can be conducted on relatively short time scales, continuous ADRs can achieve very high cooling powers with relatively low mass. The technique is particularly well-suited to systems using cryocoolers, since heat is rejected from the continuous ADR over a large fraction of the cycle period (from typically 50 to near $100 \%$ ), as opposed to short bursts of heat between long hold times for single-shot systems.

\section{2-Stage CADR}

A continuous ADR (CADR) can be assembled from as few as two stages, which can be arranged in parallel or in series, as shown in Figure 9. In the parallel case, each stage recycles as it would for use in a single-stage, single-shot system. Coordination of the cycling is done to ensure that one stage is always cold and thermally connected to the load, while the other is warm and rejecting heat. In the series case, one of the stages is permanently connected to the load, while the other cycles back and forth in temperature, spending about half the cycle absorbing heat from the cold stage (regenerating its cooling capacity) and half the cycle rejecting heat to the sink.

The parallel configuration requires two identical stages with two heat switches each, since both stages need to thermally couple and decouple (alternately) to the load and to the heat sink. Both stages also cycle across the operating range of the ADR, so both require a high field magnet to drive the cycle. In the series configuration, only the upper stage requires heat switches to couple to the lower stage and to the heat sink. In addition, the cold (continuous) stage operates only at low temperature, and therefore does not require as large a magnetic field. In operation, the lower stage absorbs heat from the load only while the upper stage is recycling, and during the remainder of the time, the upper stage absorbs 
heat both from the load and the lower stage. As a result, the lower stage needs only about half the entropy capacity as the upper stage. (It can be sized larger - for example, to economize on production costs by making both stages identical - but the larger size will not improve the ADR's performance.)
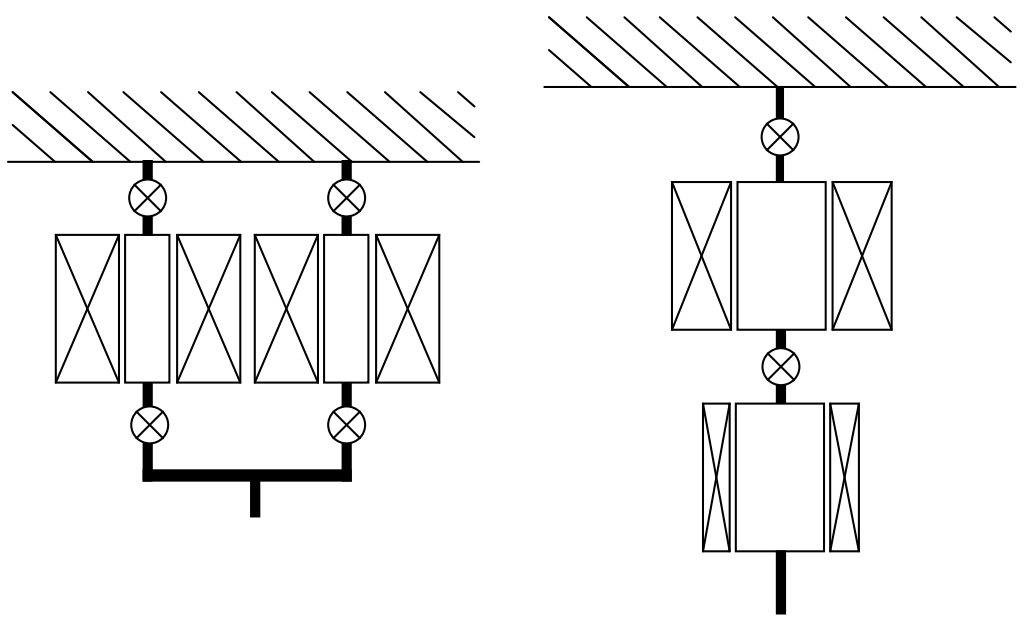

Figure 9. Two implementations of a 2-stage CADR: (a) parallel and (b) series configurations.

In both configurations, the cooling power at low temperature is dictated by the average rate at which heat is rejected at the warm end (multiplied by some efficiency factor, which can be as high as $70-80 \%$ of Carnot). In this regard, the performance of a continuous ADR is far less dependent on the size of the stages (salt mass and magnetic field) than on the rate at which they can transfer heat between stages and to the heat sink, and the times required for "overhead" operations such heat switch transitions and raising and lowering temperature between heat transfers.

The transitions between high and low temperature can be quite short, on the order of 1-2 minutes, and heat switch transitions between open and closed can also be short, a minute or less. The heat rejection time will depend on the size of the stage, and can be shortened to some extent by using larger gradients across the heat switch. In general, though, the duration is 5-10 minutes, giving a total cycle time of 20 minutes or less. These durations are largely independent of the operating temperature of the CADR, hence the cooling power tends to be a linear in temperature (Figure 10).

While both parallel and series systems have comparable efficiency, they differ by about a factor of two in cooling power because the parallel system rejects heat over close to $100 \%$ of the cycle, whereas for the series system, the fraction is only about $50 \%$. Figure 10 shows the gross cooling power of a 2-stage CADR in parallel and series configurations, using GLF stages with $3 \mathrm{~T}$ magnets and a $4.5 \mathrm{~K}$ heat sink. A 30 -minute cycle time is assumed in both cases. The net cooling power will be application specific, with reductions arising from inefficiencies in heat transfer, heat switch dissipation, eddy current losses, etc. 


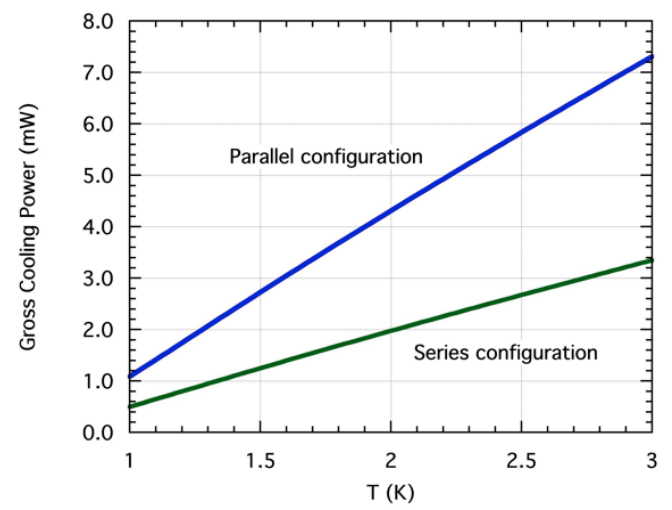

Figure 10. Gross cooling power of a 2-stage CADR in parallel and series configurations (using a $4.5 \mathrm{~K}$ heat sink).

Temperature Control - One of the key challenges for continuous ADRs is temperature control. In both configurations, the process of making and breaking thermal contact between the cycling stage(s) and the load involves changes in heat flow that tend to create temperature disturbances.

For parallel stages, the transition involves thermally connecting the recently-recycled stage before disconnecting the depleted stage. While this ensures continuous heat flow, the control system for the former begins control without an integrated demagnetization rate, with the effect that temperature will rise until the proper demagnetization rate is established. Here, the ability to calculate demagnetization rates for a given heat load can be used to significant advantage. The demagnetization rate of stage 1 before it relinquishes control can be translated into a demagnetization rate for stage 2 afterward. If the exchange of demagnetization rates is made simultaneously in both controllers, the temperature disturbance can be greatly reduced.

The situation is different for the series configuration in that the large heat capacity of the cold stage acts to buffer to minimize temperature disturbances during heat switch transitions and changing heat flows. Moreover, the process of thermally connecting the two stages can be done without any impulse by zeroing the gradient across the heat switch when it is opened and closed. The heat flow will then only gradually change as the gradient is increased and decreased, significantly reducing temperature control errors. In fact, the process can be arbitrarily slow, to achieve the desired temperature stability. The cost is cycle speed, and cooling power.

Multi-Stage CADR 
In both parallel and series configurations, stages can be added to enable operation at lower $\mathrm{T}$ or to use a warm heat sink. For parallel stages, pairs of stages and heat switches must be added. This becomes problematic for systems operating at very low temperature (less than about $300 \mathrm{mK}$ ) where heat switch technologies become the driver for system design. In particular, heat switches capable of operating at temperatures below this threshold are metallic (e.g. superconducting and magnetostrictive) and tend to have thermal conductances in the open state that are a very strong function of temperature. Hence the nearly constant heat leak through the coldest heat switches in a parallel system makes it increasingly unattractive for CADRs operating below $100 \mathrm{mK}$.

For CADRs operating below $300 \mathrm{mK}$, a series configuration is assumed, as shown in Figure 11. The specific design shown is for a 4-stage CADR operating at $50 \mathrm{mK}$ using either a normal helium bath or a cryocooler operating at up to $5 \mathrm{~K}$. Table 2 summarizes the design parameters for the full system (Figure 12) which was based on the need for $5 \mu \mathrm{W}$ of cooling power at $50 \mathrm{mK}$ for the large $\mathrm{x}$-ray microcalorimeter array proposed for the Constellation$\mathrm{X}$ mission[33].

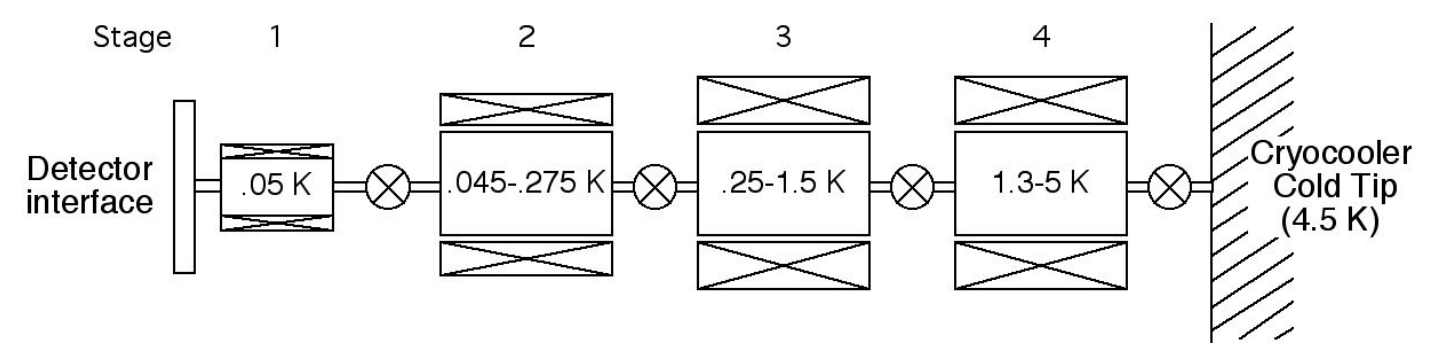

Figure 11. Schematic of a 4-stage CADR design for operation at $50 \mathrm{mK}$, using a 4-5 K heat sink. 


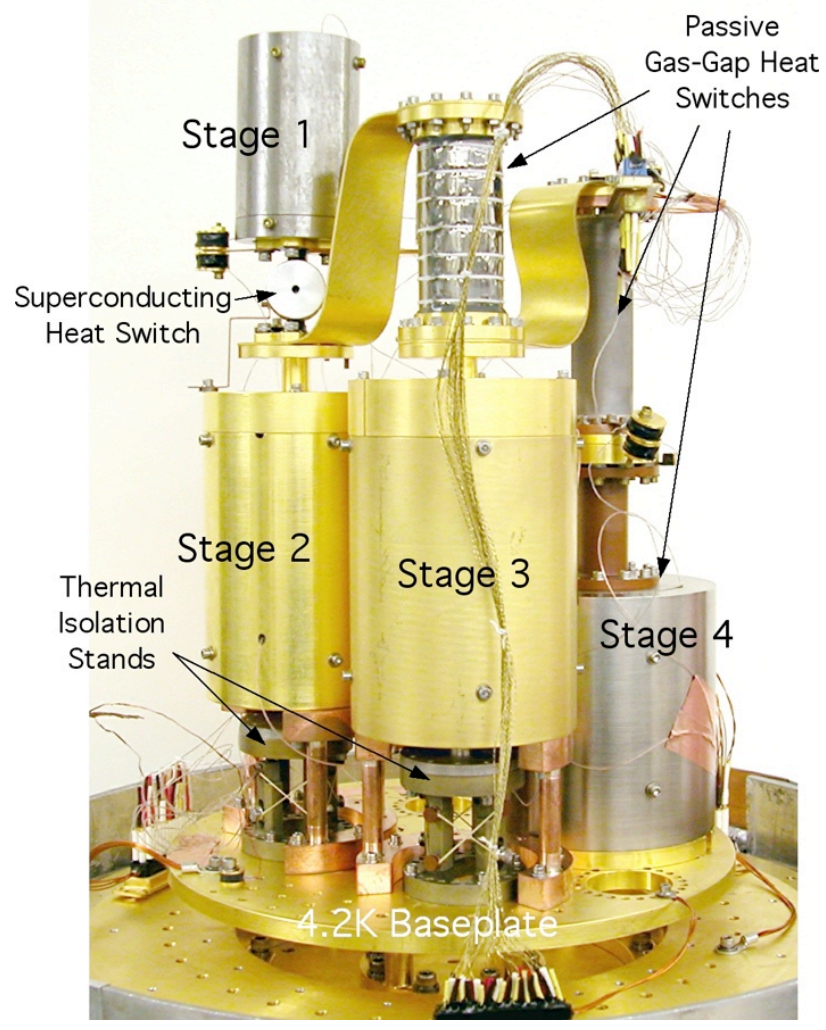

Figure 12. Complete 4-stage CADR capable of operating at $50 \mathrm{mK}$ and below, operating with a $4.2 \mathrm{~K}$ helium bath.

Table 2. Design parameters for the 4-stage CADR in Figure 13.

\begin{tabular}{|llllll|}
\hline Stage & Refrigerant & B $(\mathrm{T})$ & Thigh $(\mathrm{K})$ & Tlow $(\mathrm{K})$ & Mass $(\mathrm{kg})$ \\
\hline $\mathbf{1}$ & $60 \mathrm{~g} \mathrm{CPA}$ & 0.1 & & 0.05 & 0.5 \\
\hline $\mathbf{2}$ & $100 \mathrm{~g} \mathrm{CPA}$ & 0.5 & 0.3 & 0.045 & 1.7 \\
\hline $\mathbf{3}$ & $100 \mathrm{~g} \mathrm{CPA}$ & 1.5 & 1.3 & 0.25 & 2.4 \\
\hline $\mathbf{4}$ & $65 \mathrm{~g} \mathrm{GLF}$ & 4.0 & 5 & 1.15 & 3.1 \\
\hline
\end{tabular}

The design and optimization process follows the guidelines for single-stages, but is far more constrained by heat switches[29]. Entropy is transferred between stages at a progression of temperatures up to the heat sink. While gas-gap switches are very versatile and useful over a very wide range, at $50 \mathrm{mK}$ the choices are limited to metallic switches: mechanical, superconducting and magnetostrictive. A superconducting switch was used [29] because it is compact and relatively easy to fabricate, has very high switching ratios and modest field requirements, and (in anticipation of applications in space instruments) rugged. 
The upper stages use passive gas-gap heat switches[25]. Their design is much simpler than active versions, and eliminates the thermal connection between a separately heated getter and the switch body that can lead to significant heat leaks into the ADR stages. In addition, transitions between the open and closed states are instantaneous, allowing the stages to cycle at higher rates.

The choice of which salt to use in each stage is straightforward, and generally follows the same considerations as a for a single ADR stage. The main difference is that each stage in a CADR needs to support much larger heat fluxes - that is, larger heat flow in a smaller salt pill. Thus the salt pill requires a much more substantial thermal bus. For materials like FAA that are highly corrosive and have traditionally used gold wires for the thermal bus, the amount (and cost) of bus material is a concern. Instead, Stages 1-3 use CPA, which is not corrosive to copper, grown on to a copper thermal bus[13] machined using wire EDM techniques. Stage 4, the warmest, uses GLF.

Salt mass and magnetic field are chosen to achieve an entropy capacity in each stage that is $20-30 \%$ larger than the entropy capacity of the adjacent, colder stage. The extra capacity compensates for transfer inefficiency and parasitic heat leaks.

CADR Cycling - Operation of a multi-stage CADR requires the same coordination of heat transfers between stages, but on a larger scale. Each stage is independently temperature controlled using the magnet current, and a simple cycling algorithm is used to automate the operation to achieve a cascade of heat from Stage 1, through each upper stage, to the heat sink. Alternatively, one may view the process as a cascade of cooling capacity down to the cold stage. By orienting the control algorithm toward the latter, the system becomes quite robust, capable of starting from an arbitrary condition (such as all stages warm after cooldown), or recovering from a perturbing event, such as a change in operating temperature.

Figure 13 shows the repetitive cycling of the CADR at $50 \mathrm{mK}$. The cycle period is 20 minutes. Cooling power, as shown in Figure 15, is the maximum applied heat load at the continuous stage that can be tolerated indefinitely. Higher heat loads can be accommodated for limited periods, as long as the average is below the cooling power limit.

Figure 14 gives the efficiency of the 4-stage CADR, calculated as the ratio of entropy absorbed at the cold stage to average entropy transferred to the heat sink. It includes all internal heat loads, inefficiencies in transferring heat between stages and magnet/shield hysteresis, but does not include power dissipated by the room temperature electronics. 


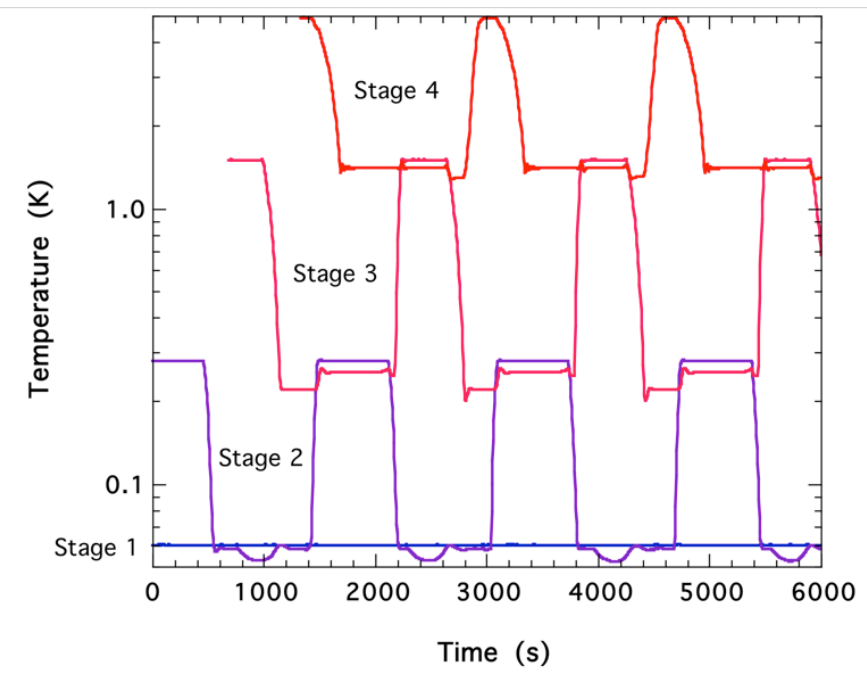

Figure 13. Cycling of a 4-stage CADR operating at $50 \mathrm{mK}$ using a $4.2 \mathrm{~K}$ heat sink.

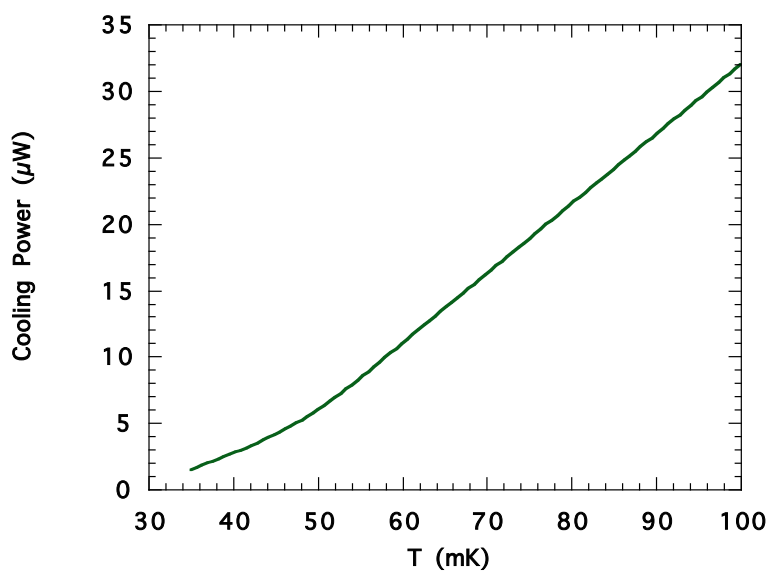

Figure 14. Cooling power of the 4-stage CADR.

\section{HYBRID ADRs}

In the same way that individual stages are the building blocks of multi-stage ADRs, groups of ADR stages can be considered the building blocks of more complex coolers. For example, Figure 15 shows a design developed for the Primordial Inflation Explorer (PIXIE)[33], an instrument designed to probe the polarization of the cosmic microwave background. The measurement involves making continuous all-sky surveys, and thus continuous cooling of the detectors and telescope is required. The 1-m scale telescope must be cooled close to the background temperature of $2.725 \mathrm{~K}$, and the detectors must be cooled to $100 \mathrm{mK}$.

The design uses a parallel 2-stage CADR to cool the telescope and a series 3-stage CADR to cool the detectors. The driver for the parallel configuration is the relatively large heat load 
on the telescope $(\sim 6 \mathrm{~mW}$ at $2.73 \mathrm{~K})$ which must be rejected to a $4.5 \mathrm{~K}$ cryocooler. The detector heat load, on the other hand, is small - a few microwatts at $100 \mathrm{mK}$ - making the series configuration more appropriate. The heat it rejects to the 2-stage CADR is a small fraction of the telescope load, so the control system can treat the two cooling chains as essentially independent.

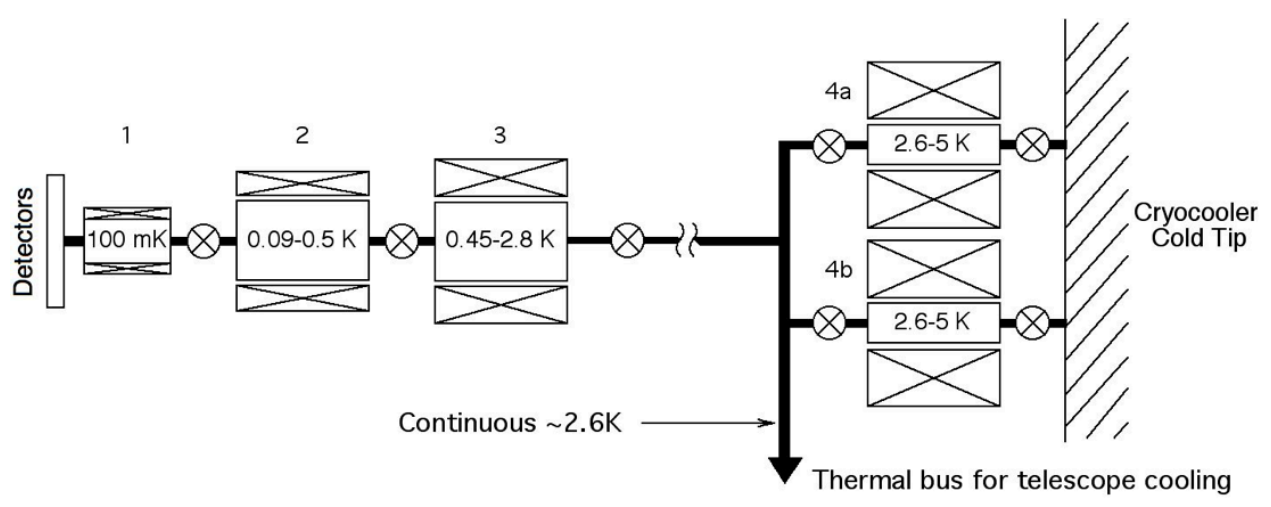

Figure 15. The 5-stage ADR designed for the PIXIE instrument.

It is also possible to combine ADRs with other refrigeration techniques. As an example, a hybrid ADR/sorption cooler has been proposed for the SAFARI instrument[34] on the Japanese-led SPICA mission[35]. The combination benefits from the flight heritage of $\mathrm{He}-3$ sorption coolers on Herschel[36] and the low mass of a single-stage ADR that operates only between $50 \mathrm{mK}$ and $300 \mathrm{mK}$. The hybrid arrangement yields a lower mass and wider operating range than either cooler by itself could achieve.

Particularly for space missions, where the allocation of system-level resources (e.g. power, volume or mass) or subsystem resources (e.g. heat load on upper-stage cryocoolers) can impose significant limitations on instrument design, hybrid coolers may at times be the only way to meet complex cooling requirements.

\section{SUMMARY}

The magnetocaloric effect is a powerful, efficient cooling mechanism that is finding applications in cooling systems all the way from room temperature to the deep sub-kelvin regime. At low temperature (helium temperature and lower), the most common implementation of the magnetocaloric effect is in ADRs. With its economy of components (a salt pill, magnet, heat switch and mechanical suspension), ADRs are easily manufactured and assembled, and easily operated through control of magnet current.

Their ability to operate at close to Carnot efficiency, the ability to control temperature losslessly over their operating range, and their modular construction, are features that make ADRs well-suited to multi-staging. Multi-staging has many benefits, including wider operating range and higher efficiency (through reductions in internal parasitic heat loads), 
and enables ADRs to be configured for continuous operation. The possible architectures for multi-stage ADRs are virtually unlimited, since ADR stages can also be combined with other refrigeration techniques (sorption coolers, for example) to create hybrid systems that take advantage of their unique characteristics.

The main driver for ADR development has been the growing use of low temperature detectors in space, but the expanded capabilities that are now possible are finding utility in laboratory systems. In particular, the trend toward the use of mechanical cryocoolers as the basis for cooling, rather than liquid helium, is producing a gap in operating temperature. Whereas helium can be pumped to as low as $1 \mathrm{~K}$, commercial cryocoolers operate only down to about $3 \mathrm{~K}$. ADRs, operating either as single-shot or continuous coolers, offer a convenient means of bridging the gap, as well as providing a basis for cooling to even lower temperature. This can be seen in the increasing availability of commercial ADRs, such as those by Janis Research Corp. [37] and HPD, Inc., [39] that are designed for cryogen-free operation.

\section{REFERENCES}

[1] Serlemitsos, A. T., SanSebastian, M., and Kunes, E., "Final Design of the XRS/Astro-E ADR," in Adv. in Cryo. Eng. 43A, edited by P. Kittel, et al., Plenum, New York, 1988, pp. 957963.

[2] R.L. Kelley, et al., The Astro-E high resolution X-Ray Spectrometer, Proceedings of the SPIE Conference on EUV, X-Ray, and Gamma-Ray Instrumentation for Astronomy X, Vol. 3765 (1999).

[3] K.D. Irwin, G.C. Hilton, D.A. Wollman, and J.M. Martinis, X-ray detection using a superconducting transition-edge sensor microcalorimeter with electrothermal feedback, Appl. Phys. Lett. 69:1945 (1996).

[4] Langevin P, Ann. Chem. Phys. 5 pp. 70 (1905)

[5] Debye P, Ann. Physik 81 pp. 1154 (1926)

[6] Giauque WF and MacDougall DP, J. Am. Chem. Soc. 57, 1175 (1935).

[7] Darby, J. et al, Proc. Phys. Soc. A64, 861 (1951).

[8] C.V. Heer, C.B. Barnes and J.G. Daunt, Rev. Sci. Instrum. 25:11 (1954).

[9] S.H. Moseley, J.C. Mather, and D. McCammon, "Thermal detectors as x-ray spectrometers", J. Appl. Phys., vol. 56, pp. 1257-1262 Sept. 1984

[10] A.T. Serlemitsos, M. SanSebastian and E. Kunes, Design of a spaceworthy adiabatic demagnetization refrigerator, Cryogenics 32:117 (1992).

[11] P.T. Timbie, G.M. Bernstein, P.L. Richards, Development of an adiabatic demagnetization refrigerator for SIRTF, Cryogenics 30, 271-275 (1990). 
[12] C. Hagmann and P.L. Richards, Two-stage magnetic refrigerator for astronomical applications with reservoir temperatures above 4K, Cryogenics 34:221 (1994).

[13] Shirron PJ, Canavan ER, DiPirro MJ, Tuttle JG, Yeager CJ. A Multi-Stage ContinuousDuty Adiabatic Demagnetization Refrigerator. Adv. Cryo. Eng. 2000;45:1629-38.

[14] F. Pobell, "Matter and Methods at Low Temperatures", $3^{\text {rd }}$ Ed., Springer-Verlag, New York, NY (2007), pp. 203-213.

[15] Lounasmaa, O. V. "Experimental Principles and Methods Below 1 K", Academic Press, London, UK (1974).

[16] 0.E. Vilches and J.C. Wheatley, Measurements of the specific heat of three magnetic salts at low temperatures, Phys. Rev. 148:509 (1966).

[17] E.W. Hornung, R.A. Fisher, G.E. Brodale and W.F. Giauque, Magnetothermodynamics of gadolinium gallium garnet, J. Chem. Phys. 61:282 (1974).

[18] Numazawa T, Kamiya K, Shirron PJ, DiPirro MJ, Matsumoto K, Magnetocaloric Effect of Polycrystal GdLiF4 for Adiabatic Demagnetization Refrigeration, AIP Conference Proceedings, 850 (2006) 1579-1580.

[19] Kelley RL, et al., "The Suzaku High Resolution X-Ray Spectrometer”, Publ. Astron. Soc. Japan 59 (2007) S77-S112.

[20] DiPirro, MJ, Shirron PJ, Tuttle JG, Canavan ER, Design and Test of Passively Operated Heat Switches for 0.2 to $15 \mathrm{~K}$, Adv. Cryo. Eng. 49 (2004) 436-442.

[21] Kurti, N., Suppl. Bull. Int. Inst. Refrig. Annexe 1955-3, (1955) p. 587.

[22] Kittel P, Temperature Stabilized Adiabatic Demagnetization for Space Applications, Cryogenics, 20, (1980), pp. 599-600.

[23] G. Bernstein, S. Labov, D. Landis, N. Madden, I. Millet, E. Silver, P. Richards, Automated temperature regulation system for adiabatic demagnetization refrigerators, Cryogenics $\mathbf{3 1}$ (1991) 99-101.

[24] C Yeager, E Maloof, S Yano, T Shimzu, Advanced adiabatic demagnetization refrigerators' temperature control system, Nuclear Instruments and Methods in Physics Research A 559 (2006) 657-659.

[25] DiPirro, MJ, Shirron PJ, Tuttle JG, Canavan ER, Design and Test of Passively Operated Heat Switches for 0.2 to 15 K, Adv. Cryo. Eng. 49 (2004) 436-442.

[26] Hagmann C and Richards PL, Specific heat of stainless steel below T = $1 \mathrm{~K}$, Cryogenics 35, 345 (1995).

[27] C. Hagmann, D.J. Benford, P.L. Richards, Paramagnetic salt pill design for magnetic refrigerators used in space applications, Cryogenics 34 213-219 (1994). 
[28] McCammon D, Shirron P, Salt Pill Design and Fabrication for Adiabatic Demagnetization Refrigerators, Cryogenics (this issue on magnetic refrigeration)

[29] Shirron P, Optimization Strategies for Single-Stage, Multi-Stage and Continuous ADRs, Cryogenics (this issue on magnetic refrigeration)

[30] C. Hagmann and P.L. Richards, Two-stage magnetic refrigerator for astronomical applications with reservoir temperatures above 4K, Cryogenics 34, 221 (1994).

[31] Hepburn, I. D.; Brockley-Blatt, C.; Coker, P.; Crofts, E.; Winter, B.; Milward, S.; StaffordAllen, R.; Hunt, R.; Brownhill, M.; Rando, N.; Linder, M., Space Engineering Model Cryogen Free ADR for Future ESA Space Missions, Adv. Cryo. Eng., AIP Conference Proceedings, Volume 710, pp. 1737-1745 (2004).

[32] Shirron P, Kimball M, Wegel D, Miller F, ADR Design for the Soft X-Ray Spectrometer Instrument on the Astro-H Mission, Cryogenics 50, 494-499 (2010).

[33] Whitehouse PL, Shirron PJ, Kelley RL, The X-ray Microcalorimeter Spectrometer (XMS): a Reference Cryogenic Instrument Design for Constellation-X, Cryogenics 44 (2004) 543-549.

[34] PJ Shirron, MO Kimball, DJ Fixsen, AJ Kogut, X Li, MJ DiPirro, Design of the PIXIE Adiabatic Demagnetization Refrigerators, Cryogenics 52, 140-144 (2011).

[35] Luchier, N. Duval, JM., Duband, L. and Tirolien, T., "Performances of the $50 \mathrm{mK}$ ADR/sorption cooler" Cryogenics, 52 (2012), pp. 152-157

[36] H Sugita, Y Sato, T Nakagawa, H Murakami, H Kaneda, K Enya, M Murakami, S Tsunematsu, M Hirabayashi, Development of mechanical cryocoolers for the Japanese IR space telescope SPICA, Cryogenics 48 (2008) 258-266

[37] Duband, L., Clerc, L., Ercolani, E., Guillemet, L. and Vallcorba, R., "Herschel flight models sorption coolers" Cryogenics 48 (2008) 95-105.

[38] Janis Research Co., Inc., 2 Jewel Dr, Wilmington, MA 01887

[39] High Performance Devices, Inc., 1668 Valtec Lane, Suite C, Boulder, CO 80301 